\title{
Bioavailability and Biokinetics of Anthocyanins From Red Grape Juice and Red Wine
}

\author{
Roland Bitsch, ${ }^{1 *}$ Michael Netzel, ${ }^{1}$ Thomas Frank, ${ }^{2}$ Gabriele Strass, ${ }^{1}$ and Irmgard Bitsch ${ }^{3}$ \\ ${ }^{1}$ Institute of Nutritional Sciences, Friedrich-Schiller-University Jena, Dornburgerstrasse 29, 07743 Jena, Germany \\ ${ }^{2}$ IMFORM GmbH International Clinical Research, Birkenweg 14, 64295 Darmstadt, Germany \\ ${ }^{3}$ Institute of Nutritional Sciences, Justus-Liebig-University Giessen, Wilhelmstrasse 20, 35392 Giessen, Germany
}

Received 3 March 2004; revised 24 May 2004; accepted 15 June 2004

\begin{abstract}
In a comparative study, 9 healthy volunteers ingested a single oral dose of $400 \mathrm{~mL}$ red grape juice or red wine with dose-adjusted anthocyanin content $(283.5 \mathrm{mg}$ or $279.6 \mathrm{mg}$, resp) in crossover. The content of anthocyanin glucosides was detected in plasma and urinary excretion. Additionally, the plasmatic antioxidant activity was assessed after intake. Based on the plasma content, biokinetic criteria of the single anthocyanins were calculated, such as AUC, $c_{\max }, t_{\max }$, and the elimination rate $\mathrm{t}_{1 / 2}$. The urinary excretion of total anthocyanins differed significantly and amounted to $0.18 \%$ (red wine) and $0.23 \%$ (red grape juice) of the administered dose. Additionally, the plasmatic antioxidant activity increased to higher levels after juice ingestion compared to wine. The intestinal absorption of the anthocyanins of red grape juice seemed to be improved compared to red wine, suggesting a possible synergistic effect of the glucose content of the juice. The improved absorption resulted in an enhanced plasmatic bioactivity.
\end{abstract}

\section{INTRODUCTION}

The health protecting effects of anthocyanins are well known as is revealed from epidemiological studies $[1,2$, $3]$. The in vitro proved antioxidative activity is the predominant characteristic of anthocyanins as for other plant phenolics and may be in relation to health benefits, protecting thus the body tissues against the oxidative damage by oxygen free radicals, which may play a role in chronic diseases.

Despite the indications of their biological activity, there is up to now only sparse information on the in vivo bioavailability and bioactivity. Moreover, biokinetic data of their absorption, distribution, metabolism, and elimination in human beings are lacking so far but are a precondition for the assessment of their efficacy in the organism. Additionally, it is controversially debated whether alcohol may affect the bioavailability and renal excretion of this group of plant phenolics.

The objective of this study is the comparative assessment of the bioavailability and bioactivity of anthocyanins of red grape juice versus red wine after ingestion.

\section{MATERIALS AND METHODS}

We recruited for the study 9 healthy volunteers (4 males and 5 females) in the age range 24 to 34 years with body mass indices from 19.7 to $26.3 \mathrm{~kg} / \mathrm{m}^{2}$. As beverages, a German red wine (Lemberger) and a commercial red grape juice concentrate were provided. In an open, single-center study under controlled conditions, the volunteers ingested after an overnight fasting a single portion of either $400 \mathrm{~mL}$ red wine (variety Lemberger, Germany) or the same volume of diluted commercially obtained red grape juice concentrate with identical total anthocyanin doses of $283.5 \mathrm{mg}$ (red grape juice) or $279.6 \mathrm{mg}$ (red wine). The test phases were separated by a 4 -week interval. Each person served as his or her own control. The order of the beverage ingestion was not randomized. The wine and the juice were ingested together with white rolls and $30 \mathrm{~g}$ of cheese. The volunteers were instructed not to consume any anthocyanin-containing foods or juices or alcohol 24 hours before and during the study.

Venous blood samples were taken initially (predose as baseline) and at $0.25,0.50,1.0,1.5,2$, and 3 hours after intake of juice or wine, respectively. Urine samples were additionally collected predose and quantitatively in hourly intervals over a period of 7 hours. The study design was approved by the Ethical Commission of the University of Giessen, Germany.

In plasma and urine, the anthocyanin content was analyzed by the usual HPLC methods $[4,5,6,7]$. In brief description, after extraction from plasma by using an ODS solid phase extraction cartridge and elution with $5 \mathrm{~mL}$ $0.44 \mathrm{M}$ trifluoruacetic acid (TFA) in methanol, the anthocyanins were separated using a Prontosil Eurobond RP-18 column protected by a LiChrospher $100 \mathrm{RP}-18$ guard column and isocratic elution with water/acetonitrile/formic acid (81/10/9, pH 1.6) as mobile phase. Single substances were detected at $520 \mathrm{~nm}$ with a photodiode array detector 
TABLE 1. Ingested bioactive compounds.

\begin{tabular}{lcc}
\hline Component/parameter & $\begin{array}{c}\text { Red grape juice conc. } \\
\text { diluted }(400 \mathrm{~mL})\end{array}$ & Red wine $(400 \mathrm{~mL})$ \\
\hline Antioxidant capacity by & 6.0 & 8.6 \\
TRAP assay (mmol) & 283.5 & 279.6 \\
Total anthocyanins (mg) & 15.2 & 74.8 \\
Flavan-3-ols (mg) & 5.6 & 2.4 \\
Flavonols (mg) & 9.2 & 6.8 \\
Resveratrols (mg) & 16.8 & 23.2 \\
Phenolic acids (mg) & 330.3 & 386.8 \\
\hline Total phenolics $(\mathrm{mg})$ & & \\
\hline
\end{tabular}

and a UV-VIS detector. Identity of the separated substances was ensured by comparing their retention time and UV-VIS spectra with those of commercially available standards. The detection limits of the analyzed anthocyanins were between $0.65 \mathrm{ng} / \mathrm{mL}$ (cyanidin-3-glucoside) and $5.2 \mathrm{ng} / \mathrm{mL}$ (malvidin-3-glucoside) in all matrices. For calibration (average $\mathrm{r}^{2}$ value 0.998), blank urine and plasma samples were spiked with known concentrations of standard solutions.

The chromatographic conditions were identical for beverages, plasma, and urine. Juice and red wine samples were diluted $1: 50$ with HPLC mobile phase and centrifuged before injection into the HPLC column. The analyzed anthocyanin profile in grape juice differed slightly from that in wine in a lower content of glucosides of petunidin (42 versus $91 \mu \mathrm{g} / \mathrm{mL}$ ) and malvidin (327 versus $461 \mu \mathrm{g} / \mathrm{mL})$ and a higher glucoside content of delphinidin (124 versus $95 \mu \mathrm{g} / \mathrm{mL}$ ) and peonidin (208 versus $45 \mu \mathrm{g} / \mathrm{mL}$ ). In predose plasma and urine samples of the volunteers, the anthocyanin levels were below the detection limit.

Total polyphenolics were determined according to [8] by a modification of the Folin-Ciocalteau method. Conjugated polyphenols were hydrolyzed with $1 \mathrm{M}$ hydrochloric acid $(\mathrm{HCl})$ and polyphenol-lipid links broken with $2 \mathrm{M} \mathrm{NaOH}$ in $75 \%$ methanol followed by precipitation of plasma proteins with $0.75 \mathrm{M}$ metaphosphoric acid. The polyphenols were, after centrifugation, extracted from the supernatant with acetone/water (1/1) and assayed with the Folin-Ciocalteau reagent. Results were given as milligram gallic acid equivalents (GAE) per liter. The anthocyanin glucoside values in plasma and urine were based for the calculation of the pharmacocinetics/biokinetics, such as the maximal concentration $\mathrm{c}_{\max }$, its peaking time $t_{\max }$, the area under the curve AUC, and the elimination half-life $t_{1 / 2}$ [4]. Noncompartmental pharmacokinetic evaluation according to standard methods was performed [9] by using the WinNonlin Professional software (version 3.3, Pharsight Co, Mountain View, Calif). The extent and the relative bioavailability (wine versus juice) was tested for equivalence by calculating the $90 \%$ confidence interval (CI) on the basis of a one-sample $t$ test of the log-transformed intraindividual differences in dose-normalized data. Distributional assumptions were confirmed by the Shapiro-Wilk test prior to performing the $t$ test, which was based on the assumption of lognormally distributed intraindividual differences. Significant differences were accepted with $P$ values less than or equal to .05 .

The bioactivity of the phenolics in juice and wine was estimated by measuring the antioxidant activity in plasma samples with the aid of the commonly used TRAP assay according to [10]. The total phenolics ingested are shown in Table 1.

\section{RESULTS}

The pharmacokinetics of the single anthocyanins in plasma after red grape juice and red wine intake are shown in Table 2. It is noteworthy that the anthocyanins in plasma as well as in urine were nearly exclusively detected as glucosides, as was also demonstrated by others $[11,12,13,14]$. No other conjugated or free forms could be identified in plasma; merely, in the urinary excretion, two additional small peaks of unidentified metabolites appeared after juice and wine intake. The following data are therefore related to the anthocyanin glucosides. After grape juice ingestion the geometric mean $c_{\max }$ of total anthocyanins of about $100 \mathrm{ng} / \mathrm{mL}$ was estimated at a peaking time $t_{\max }$ of half an hour, whereas after red wine intake a lower $c_{\max }$ of about $43 \mathrm{ng} / \mathrm{mL}$ was reached only at a peaking time $t_{\max }$ of 1.5 hours on average. The geometric mean plasmatic AUC attained, after wine intake, only $60 \%$ of the level after grape juice. But because of the higher interindividual variability of the AUC and $c_{\max }$ after red grape juice, no statistically significant differences of the plasmatic total anthocyanin levels between the two drinks could be ascertained. Only the values of cyanidin and delphinidin glucosides were without any doubt enhanced after grape juice drinking because the concentration of both anthocyanins after wine consumption was below the detection limit.

The plasma profile of the absorbed substances was also reflected in the urinary excretion pattern of the 
TABLE 2. Plasma pharmacokinetic parameters of anthocyanins following administration of red grape juice and red wine.

\begin{tabular}{llccrr}
\hline & Anthocyanin & $\mathrm{c}_{\max }(\mathrm{ng} / \mathrm{mL})$ & $\mathrm{t}_{\max }(\mathrm{h})^{\mathrm{a}}$ & $\mathrm{AUC}(0-3)\left(\mathrm{ng}^{*} \mathrm{~h}^{*} \mathrm{~mL}^{-1}\right)$ & $\mathrm{t}_{1 / 2}(\mathrm{~h})$ \\
\hline & Cya-3-gluc & $0.42(118)$ & $0.5(0.5-0.5)$ & $0.60(82.9)$ & $1.61(22.7)$ \\
& Del-3-gluc & $6.12(66.7)$ & $0.5(0.5-1.0)$ & $11.9(55.0)$ & $1.72(26.6)$ \\
Red grape juice & Malv-3-gluc & $48.8(87.6)$ & $0.5(0.5-1.0)$ & $71.7(60.4)$ & $1.50(34.4)$ \\
& Peo-3-gluc & $27.3(51.0)$ & $0.5(0.5-0.5)$ & $49.7(36.2)$ & $1.63(19.4)$ \\
& Pet-3-gluc & $16.1(40.5)$ & $0.5(0.5-0.5)$ & $31.5(30.5)$ & $1.68(48.8)$ \\
& Total anthocyanins ${ }^{g}$ & $100.1(64.2)$ & $0.5(0.5-1.0)$ & $168.4(42.3)$ & $1.83(28.5)$ \\
\hline Red wine & Cya-3-gluc & $\mathrm{NA}$ & $\mathrm{NA}$ & $\mathrm{NA}$ & $\mathrm{NA}$ \\
& Del-3-gluc & $\mathrm{NA}$ & $\mathrm{NA}$ & $40.4(21.2)$ & $\mathrm{NA}$ \\
& Malv-3-gluc & $18.5(24.0)$ & $1.5(1.0-1.5)$ & $30.7(17.7)$ & $1.80(31.9)$ \\
& Peo-3-gluc & $12.6(16.1)$ & $1.5(1.0-1.5)$ & $29.1(15.1)$ & $1.83(40.1)$ \\
& Pet-3-gluc & $12.3(16.2)$ & $1.5(0.5-1.5)$ & $100.8(14.4)$ & $2.15(22.9)$ \\
& Total anthocyanins ${ }^{g}$ & $42.9(16.0)$ & $1.5(1.0-1.5)$ & & $1.99(28.1)$ \\
\hline
\end{tabular}

${ }^{a}$ Median (range).

gSum of cyanidin 3-glucoside, delphinidin 3-glucoside, malvidin 3-glucoside, peonidin 3-glucoside, and petunidin 3-glucoside.

NA = not applicable (concentrations below LOQ).

Data are geometric means (with geometric coefficients of variation (\%) in parentheses).

anthocyanin glucosides (Table 3). The maximal excretion rate $\mathrm{R}_{\max }$ could be detected between 1.5 and 2.5 hours after intake. This is consistent with observations of Miyazawa et al [5] and Matsumoto et al [11]. Despite the high variation also seen with the grape juice anthocyanin excretion pattern, differences between both beverages in the excreted total amount were statistically significant, reaching $0.23 \%$ of the administered dose after grape juice and $0.18 \%$ after wine ingestion with petunidin and peonidin glucoside as the highest rates and cyanidin glucoside as the lowest-percentage excretion rate in both cases. It should however be mentioned that the cumulative excreted amounts do not correspond exactly to the absorption rate. Biliary secretion, for example, may also contribute to the elimination process of some substances.

The content of total polyphenolics in plasma increased after intake of both beverages with a $c_{\max }$ after about 30 minutes (Figure 1). In contrast to plasmatic anthocyanins, the $c_{\max }$ value after grape juice intake significantly exceeded the analogous value after wine intake, indicating that flavonoids other than anthocyanins may be better absorbed. It should be noted that this increase occurred on the basis of a relatively high GAE fasting level that may be accounted for by other reducing substances (SH-compounds, ascorbic and uric acids, etc) reacting also with the modified Folin-Ciocalteau method as was described by other authors [8].

Besides the anthocyanin levels in plasma and urine, we measured the plasmatic antioxidative capacity as biomarker for the physiological efficiency of all polyphenolics that were absorbed after beverage consumption. The assessed TRAP values were adjusted to the ascorbic acid and uric acid content of the plasma to avoid any misinterpretation. As is evident from Figure 2 and Table 4, both of the beverages were able to enhance the plasmatic

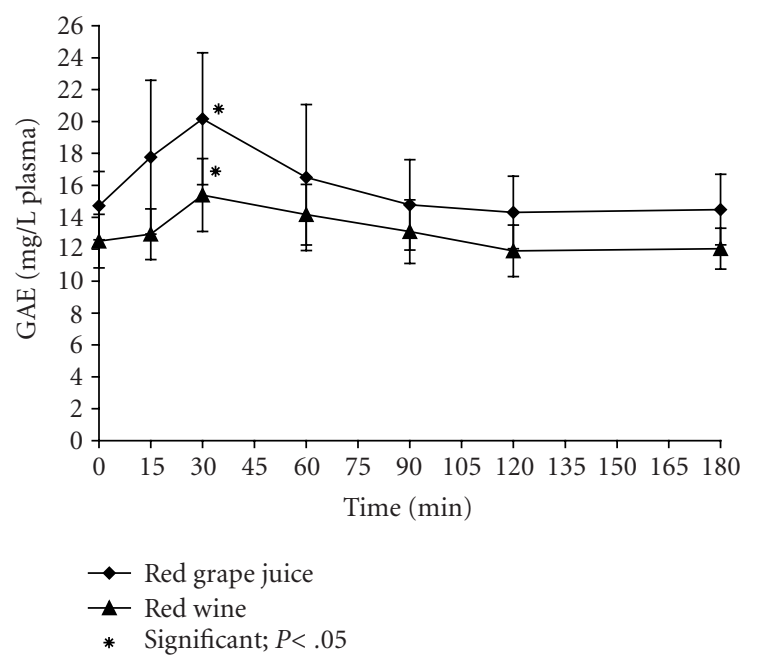

FIGURE 1. Total phenolics in plasma.

antioxidant activity, peaking between 50 and 63 minutes and dropping to initial values again after 2 hours. As with the plasmatic polyphenols, the TRAP value biokinetics over the time after grape juice intake surpassed the level after wine intake with statistical significance, suggesting a superior bioactivity of the anthocyanins and other polyphenolics and/or their metabolites from red grape juice compared to red wine.

\section{DISCUSSION}

In this comparative study, we tested the bioavailability of anthocyanins as the valuable components in red grape juice and red wine in human beings. From the 
TABLE 3. Urinary pharmacokinetic parameters of anthocyanins following administration of red grape juice and red wine. $\left(R_{\max }=\right.$ maximal observed excretion rate; $\mathrm{Ae}(0-7)=$ total amount of excreted anthocyanins; $\mathrm{Xe}(0-7)=$ percentage of excreted dose.)

\begin{tabular}{|c|c|c|c|c|c|}
\hline & Anthocyanin & $\mathrm{R}_{\max }(\mu \mathrm{g} / \mathrm{h})$ & $\mathrm{t}_{\max }, \mathrm{R}(\mathrm{h})^{\mathrm{a}}$ & Ae $(0-7)(\mu \mathrm{g})$ & $\mathrm{Xe}(0-7)(\%)$ \\
\hline \multirow{6}{*}{ Red grape juice } & Cya-3-gluc & $1.26(75.8)$ & $0.5(0.5-1.5)$ & $2.88(72.1)$ & 0.09 \\
\hline & Del-3-gluc & $39.6(91.1)$ & $0.5(0.5-1.5)$ & $101.9(72.5)$ & 0.20 \\
\hline & Mal-3-gluc & 86.7 (126) & $0.5(0.5-1.5)$ & $236.3(95.7)$ & 0.18 \\
\hline & Peo-3-gluc & $86.0(79.1)$ & $0.5(0.5-1.5)$ & $240.5(65.8)$ & 0.29 \\
\hline & Pet-3-gluc & $20.2(45.8)$ & $0.5(0.5-1.5)$ & $53.4(31.1)$ & 0.32 \\
\hline & Total anthocyanins & $241.4(82.3)$ & $0.5(0.5-1.5)$ & $653.6(66.6)$ & 0.23 \\
\hline \multirow{6}{*}{ Red wine } & Cya-3-gluc & $0.66(117)$ & $2.5(1.5-4.5)$ & $1.45(67.3)$ & 0.06 \\
\hline & Del-3-gluc & $14.9(51.5)$ & $0.5(0.5-1.5)$ & 47.7 (26.9) & 0.12 \\
\hline & Mal-3-gluc & $60.2(33.4)$ & $1.5(1.5-2.5)$ & $206.8(31.7)$ & 0.11 \\
\hline & Peo-3-gluc & $44.1(43.7)$ & $0.5(0.5-1.5)$ & $151.5(31.5)$ & 0.84 \\
\hline & Pet-3-gluc & $20.5(52.5)$ & $1.5(1.5-1.5)$ & $66.4(47.5)$ & 0.18 \\
\hline & Total anthocyanins & $137.6(29.2)$ & $1.5(1.5-2.5)$ & $491.0(19.8)$ & 0.18 \\
\hline
\end{tabular}

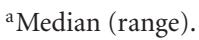

Data are geometric means (with geometric coefficients of variation (\%) in parentheses).

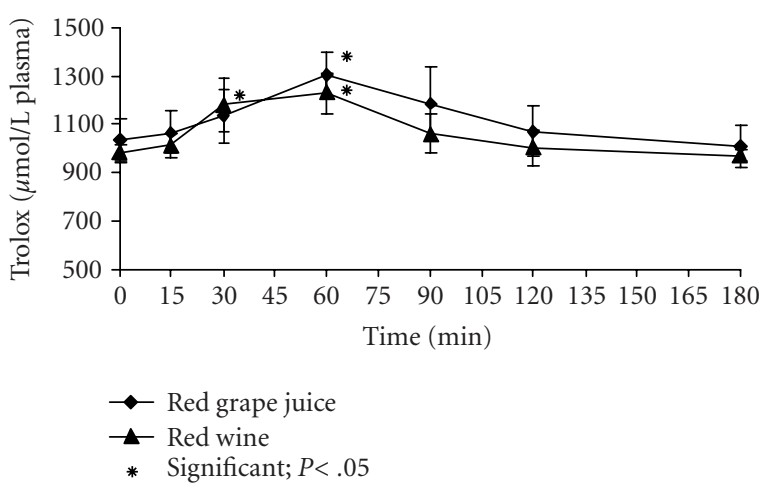

FIGURE 2. Plasmatic TRAP values after ingestion of red grape juice and red wine.

well-known French paradox, a health protective effect of the polyphenol-rich red wine had been suggested, assuming that the intestinal absorption of anthocyanins may be relieved by the alcohol content of the wine [15]. But exact information on the bioavailability of anthocyanins from alcoholic and nonalcoholic beverages is lacking so far. Moreover, kinetic data on the absorption and elimination rates of those polyphenolics may be of relevance, too, in terms of the suggested health protection.

A first proof that anthocyanins appear in plasma as intact glucosides after oral ingestion had been furnished by Tsuda et al in animals [13]. In the meantime, it could be demonstrated by our group and other authors that besides monoglucosides, higher condensed glycosylated forms in fruit juices were also incorporated from the intestine into the blood of rats and humans and are excreted in urine as such $[4,5,6,11,12,14,16,17,18,19,20]$.

The generally low plasma and urine concentrations in this study suggest that the small absorbed anthocyanin dose is subjected to a rapid metabolism. Also a decompostion of anthocyanins cannot be excluded, since Tsuda et al [13] detected protocatechuic acid in the plasma of rats fed cyanidin-3-glucoside but in a 100-fold higher dose per $\mathrm{kg}$ body weight than in the present study. As the percentage of the excreted cyanidin glucoside in the present study was by far lower than for the other anthocyanins, this may indicate that cyanidin in vivo serves as precursor and is methylated to peonidin as was already proved in rats [13] (Table 3, Xe (0-7)). The excreted total anthocyanin amount is in contrast to the values of Lapidot et al [21] who assessed a more than 10-fold excretion rate after red wine intake with a comparable anthocyanin dose but within 12 hours. Bub et al [20], on the other hand, found less than $0.03 \%$ of the ingested dose in the urine.

Even though the plasma biokinetics revealed no statistical differences between the beverages, the analogous values in the urinary excretion (Table $3, \mathrm{R}_{\max }$, Ae (0-7) and $\mathrm{Xe}(0-7))$ suggest a higher bioavailability of anthocyanins from red grape juice compared to red wine. Perhaps the observation period was relatively short to recognize bioequivalence in plasma kinetics, too. Nevertheless, it has also been considered that due to absorption on plasma proteins, the analytical recovery rate of anthocyanins may be lowered. Murkovic et al [14] calculated, from spiked plasma samples, a recovery rate of elderberry anthocyanins to only $20 \%$.

The identical elimination rates $\left(t_{1 / 2}\right)$ from plasma corresponding with a (nonstatistical) tendency to lower $\mathrm{c}_{\max }{ }^{-}$ and higher $t_{\max }$-values after red wine intake lead to the conclusion that either the anthocyanin absorption from red wine was inhibited or the intestinal uptake from red grape juice was enhanced (Table 2). Up to now, there are contradictory findings about the effect of alcohol on the gastrointestinal function and absorption of flavonoids. 
TABLE 4. TRAP biokinetics in plasma of 9 volunteers.

\begin{tabular}{cccccc}
\hline & $\begin{array}{c}c_{\max } \\
\mu \mathrm{mol}^{*} \mathrm{~L}^{-1}\end{array}$ & $\begin{array}{c}\mathrm{c}_{\max } / \mathrm{Dose} \\
\mu \mathrm{mol}^{*} \mathrm{~L}^{-1 *} \mu \mathrm{mol}^{-1}\end{array}$ & $\mathrm{t}_{\max } \min$ & $\begin{array}{c}\mathrm{AUC}(0-180) \\
\mathrm{mmol}^{*} \mathrm{~min}^{-1 *} \mathrm{~L}^{-1}\end{array}$ & $\begin{array}{c}\text { AUC }(0-180) / \mathrm{Dose} \\
\mathrm{mmol}^{*} \mathrm{~min}^{-1 *} \mathrm{mmol}^{-1}\end{array}$ \\
\hline $\begin{array}{c}\text { Red wine } \\
\text { Red grape juice }\end{array}$ & $1233 \pm 85$ & $0.143 \pm 0.010$ & $50 \pm 15$ & $192 \pm 8$ & $22.31 \pm 0.95$ \\
$S$ & $1333 \pm 62$ & $0.222 \pm 0.010$ & $63 \pm 10$ & $202 \pm 11$ & $33.72 \pm 1.86$ \\
\hline
\end{tabular}

Data are arithmetic means \pm SD.

Levanon et al [22] suggested that white wine may inhibit the postprandial contractions and thus delay the absorption of sugars and glucosides. Bub et al [20] found no difference in the absorption of malvidin-3-glucoside between red wine and dealcoholized red wine, but found a delayed bioavailability after red grape juice intake. The latter finding is in contrast to our results. Gee et al [23] and Hollman [24] could demonstrate that quercetin glucosides may interact with the intestinal glucose transporter SGLT-1, resulting in an elevated intestinal uptake. More recently, it has been shown that the intestinal lactase-phlorizin hydrolase (LPH) in the apical membrane of intestine epithelial cells and the cytosolic $\beta$-glucosidase seems to play an important role in the absorption and metabolism of flavonoids $[25,26]$. Considering the sugar content of the administered red grape juice and the similar structural features to quercetin, it is conceivable that the anthocyanins are absorbed via cotransport with the intestinal brush border SGLT-1, elevating thus the anthocyanin uptake compared to red wine [27].

This assumption is corroborated by the significantly enhanced biokinetics of the plasmatic polyphenolics after grape juice intake (Figure 1). The superior bioactivity of red grape juice compared to red wine is not at least visible in the antioxidant capacity of the plasma. The $c_{\max }$ as well as the AUC of the measured TRAP values in plasma after grape juice ingestion significantly exceeded the values after wine intake (Table 4).

\section{CONCLUSION}

The results of the present study suggest that red grape anthocyanins are absorbed in small amounts and renally excreted as intact glucosides. Red grape juice may be quite equivalent or even superior to red wine with a comparable content of bioactive flavonoids/anthocyanins. Against past opinion, the strongly antioxidative anthocyanins are obviously better absorbed from grape juice than from wine. Consequently, the plasmatic antioxidative capacity (TRAP assay) in man is stronger elevated after grape juice consumption compared to wine. Finally, from the results it is hypothesized that the anthocyanin absorption may be improved by the glucose content of the grape juice; a potential alcohol effect on the intestinal uptake and metabolism remains to be elucidated. Nevertheless, further components evolving during wine processing may interfere with the anthocyanin absorption. In future studies, more exact data on the in vivo metabolism of glucosidically bound anthocyanins and other conjugates may substantiate their health protective effects.

\section{REFERENCES}

[1] Steinmetz KA, Potter JD. Vegetables, fruit, and cancer prevention: a review. J Am Diet Assoc. 1996;96 (10):1027-1039.

[2] Prior RL. Fruits and vegetables in the prevention of cellular oxidative damage. Am J Clin Nutr. 2003;78 (suppl 3):570S-578S.

[3] Hertog MG, Feskens EJ, Hollman PC, Katan MB, Kromhout D. Dietary antioxidant flavonoids and risk of coronary heart disease: the Zutphen elderly study. Lancet. 1993;342(8878):1007-1011.

[4] Frank T, Netzel M, Strass G, Bitsch R, Bitsch I. Bioavailability of anthocyanidin-3-glucosides following consumption of red wine and red grape juice. Can J Physiol Pharmacol. 2003;81(5):423-435.

[5] Miyazawa T, Nakagawa K, Kudo M, Muraishi K, Someya K. Direct intestinal absorption of red fruit anthocyanins, cyanidin-3-glucoside and cyanidin3, 5-diglucoside, into rats and humans. J Agric Food Chem. 1999;47(3):1083-1091.

[6] Netzel M, Strass G, Janssen M, Bitsch I, Bitsch R. Bioactive anthocyanins detected in human urine after ingestion of blackcurrant juice. J Environ Pathol Toxicol Oncol. 2001;20(2):89-95.

[7] Netzel M, Strass G, Kaul C, Bitsch I, Dietrich H, Bitsch R. In vivo antioxidative capacity of a composite berry juice. Food Res Int. 2002;35(2-3):213-216.

[8] Serafini M, Maiani G, Ferro-Luzzi A. Alcohol-free red wine enhances plasma antioxidant capacity in humans. J Nutr. 1998;128(6):1003-1007.

[9] Cawello W. Parameters for Compartment-Free Pharmacokinetics. Aachen: Shaker Verlag; 1999.

[10] Ghiselli A, Serafini M, Maiani G, Azzini E, FerroLuzzi A. A fluorescence-based method for measuring total plasma antioxidant capability. Free Radic Biol Med. 1995;18(1):29-36.

[11] Matsumoto $H$, Inaba $H$, Kishi $M$, Tominaga S, Hirayama M, Tsuda T. Orally administered delphinidin-3-rutinoside and cyanidin-3-rutinoside are directly absorbed in rats and humans and appear in the blood as the intact forms. J Agric Food Chem. 2001;49(3):1546-1551. 
[12] Cao G, Muccitelli HU, Sanchez-Moreno C, Prior RL. Anthocyanins are absorbed in glycated forms in elderly women: a pharmacokinetic study. Am J Clin Nutr. 2001;73(5):920-926.

[13] Tsuda T, Horio F, Osawa T. Absorption and metabolism of cyanidin-3-O-beta-D-glucoside in rats. FEBS Lett. 1999;449(2-3):179-182.

[14] Murkovic M, Adam U, Pfannhauser W. Analysis of anthocyane glycosides in human serum. Fresenius $J$ Anal Chem. 2000;366(4):379-381.

[15] Renaud S, de Lorgeril M. Wine, alcohol, platelets, and the French paradox for coronary heart disease. Lancet. 1992;339(8808):1523-1526.

[16] Netzel M, Strass G, Carle E, et al. Schwarzer Johannisbeersaft $=$ functional food? Lebensmittelchemie. 2000;54:84-85.

[17] Mülleder U, Murkovic M, Pfannhauser W. Urinary excretion of cyanidin glycosides. J Biochem Biophys Methods. 2002;53(1-3):61-66.

[18] Wu X, Cao G, Prior RL. Absorption and metabolism of anthocyanins in elderly women after consumption of elderberry or blueberry. J Nutr. 2002;132 (7):1865-1871.

[19] Murkovic M, Mülleder U, Adam U, Pfannhauser W. Detection of anthocyanins from elderberry juice in human urine. J Sci Food Agric. 2001;81(9):934-937.

[20] Bub A, Watzl B, Heeb D, Rechkemmer G, Briviba K. Malvidin-3-glucoside bioavailability in humans after ingestion of red wine, dealcoholized red wine and red grape juice. Eur J Nutr. 2001;40(3):113-120.

[21] Lapidot T, Harel S, Granit R, Kanner J. Bioavailability of red wine anthocyanins as detected in human urine. J Agric Food Chem. 1998;46(10):4297-4302.

[22] Levanon D, Goss B, Chen JDZ. Inhibitory effect of white wine on gastric myoelectrical activity and the role of vagal tone. Dig Dis Sci. 2002;47(11):25002505.

[23] Gee JM, DuPont MS, Day AJ, Plumb GW, Williamson G, Johnson IT. Intestinal transport of quercetin glycosides in rats involves both deglycosylation and interaction with the hexose transport pathway. J Nutr. 2000;130(11):2765-2771.

[24] Hollman PCH. Evidence for health benefits of plant phenols: local or systemic effects? J Sci Food Agric. 2001;81(9):842-852.

[25] Day AJ, Canada FJ, Diaz JC, et al. Dietary flavonoid and isoflavone glycosides are hydrolysed by the lactase site of lactase phlorizin hydrolase. FEBS Lett. 2000;468(2-3):166-170.

[26] Németh K, Plumb GW, Berrin JG, et al. Deglycosylation by small intestinal epithelial cell $\beta$-glucosidases is a critical step in the absorption and metabolism of dietary flavonoid glycosides in humans. Eur J Nutr. 2003;42(1):29-42.
[27] Ferraris RP. Dietary and developmental regulation of intestinal sugar transport. Biochem J. 2001;360(pt 2):265-276.

\footnotetext{
* Corresponding author.

E-mail: roland.bitsch@uni-jena.de

Fax: +49 3641 949632; Tel: +493641949630
} 

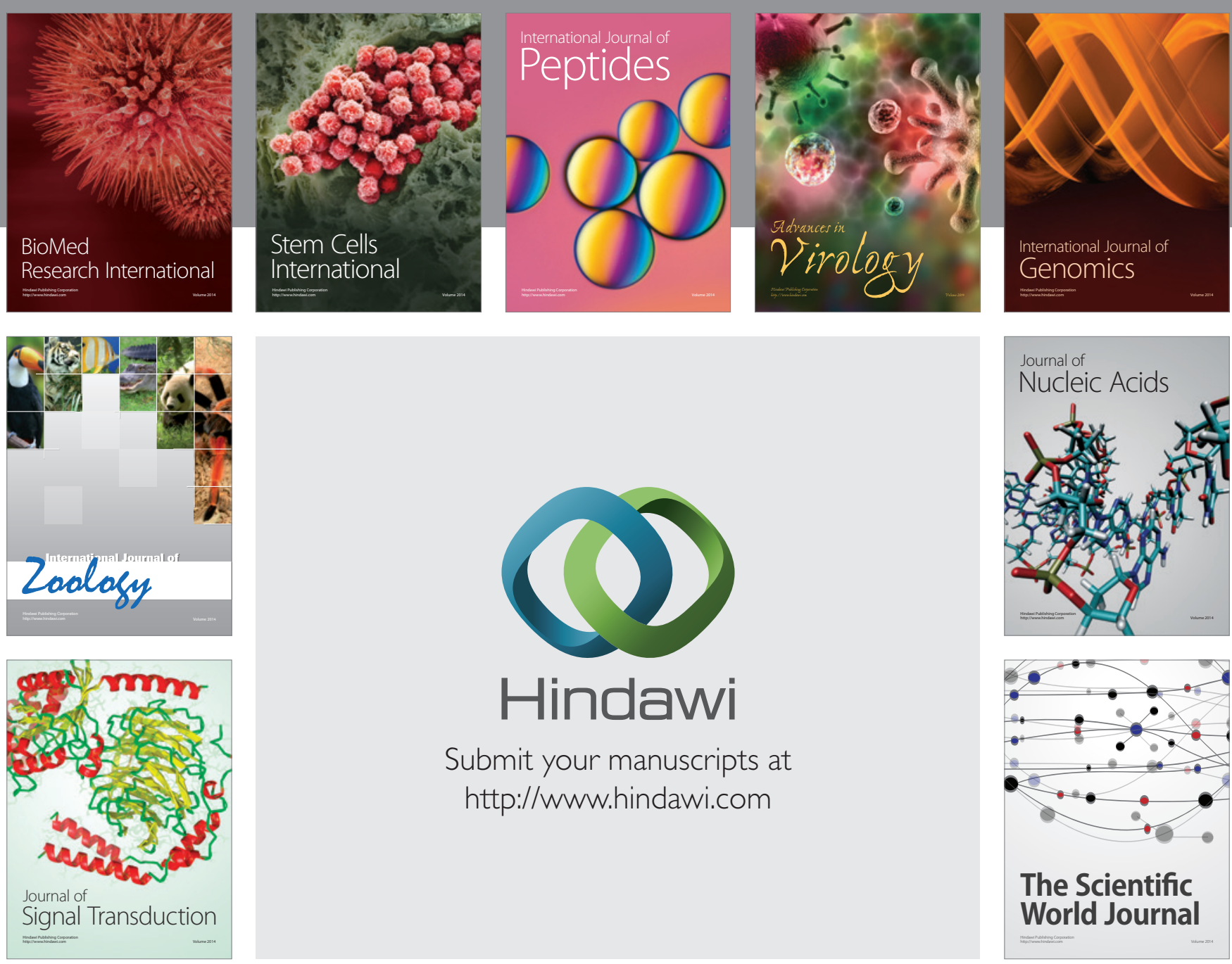

Submit your manuscripts at

http://www.hindawi.com
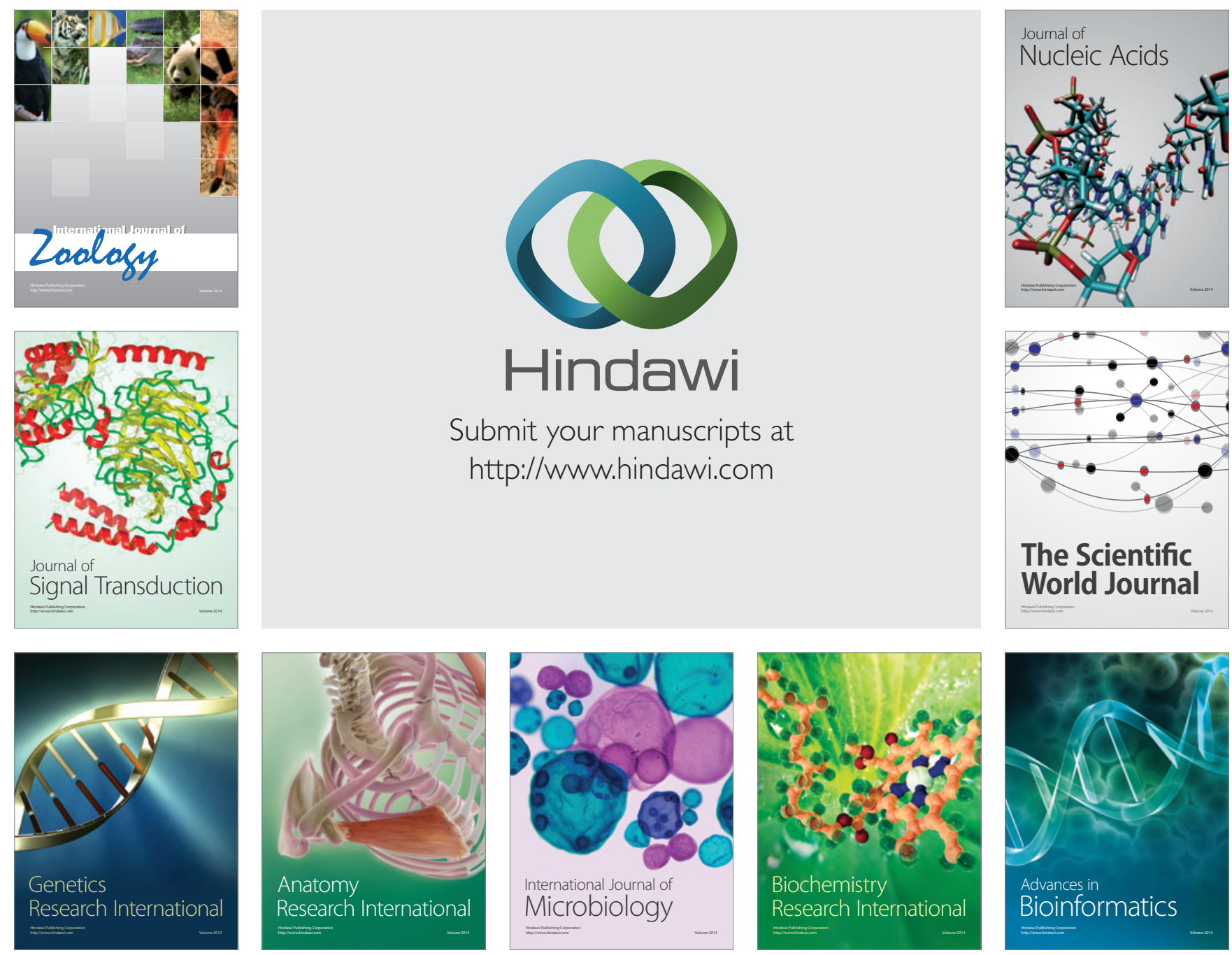

The Scientific World Journal
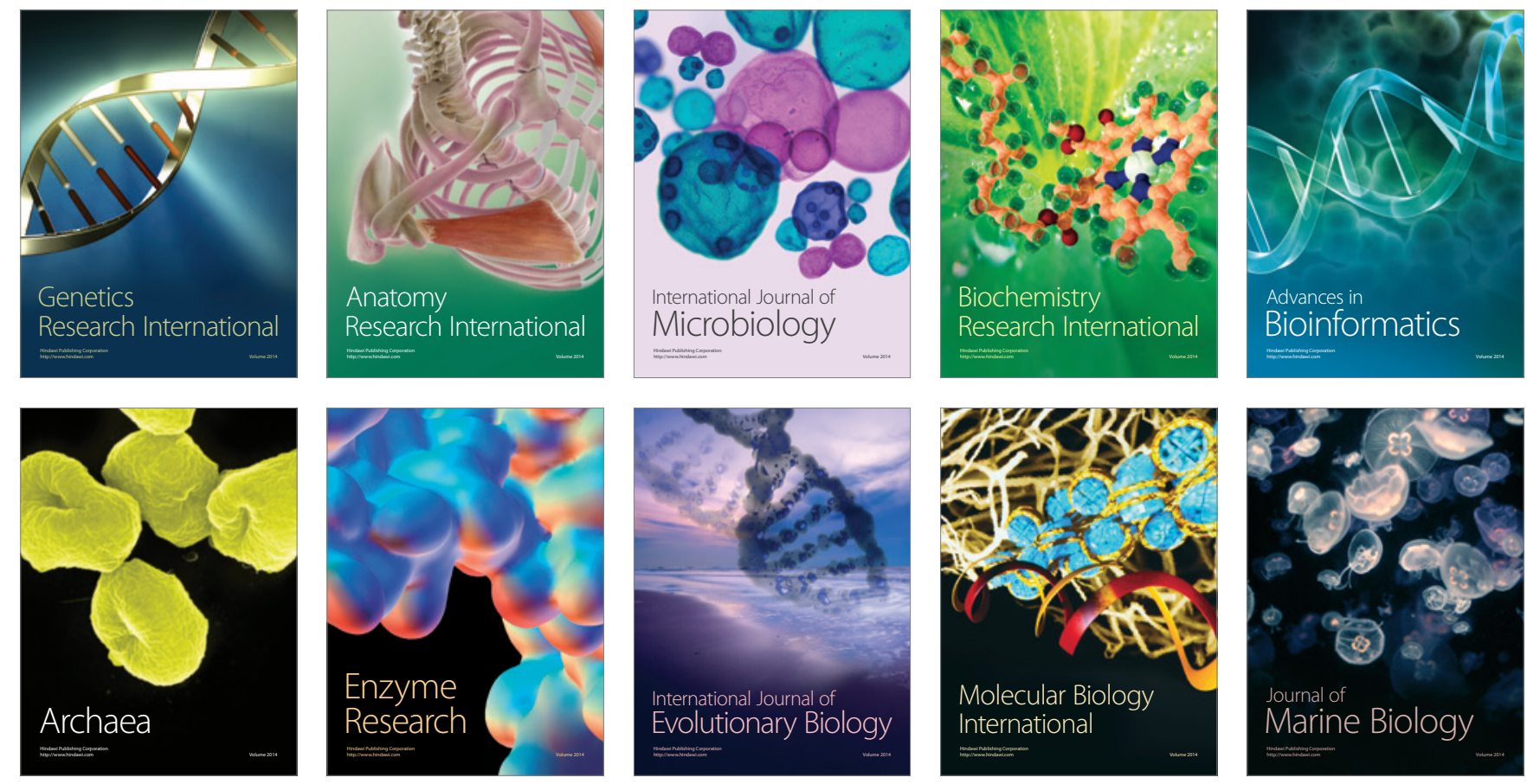